DOI: 10.35218/armca.2021.1.08

\title{
Art roman et art moderne : « affinités sentimentales »
}

\author{
Valérie Buisine
}

\begin{abstract}
The history of art in the twentieth century reveals many and varied affinities with roman art, both formal and spiritual, especially for the painters of the Second School of Paris and the non-figuration. However, it would be more correct to speak of "sentimental affinities" because this story features artists and thinkers for whom the essential stake is to renew links with the public, to nourish modern man from within in a world to be rebuilt.
\end{abstract}

Keywords: roman art ; abstract art ; non-figurative art ; Second School of Paris; spirituality ; sacred art

\section{Plan de l'article}

- Recherche d'une voie propre

- Le rôle de l'art roman

- Albert Gleizes et un cubisme « à la romane »

- L'art roman s'expose

- La peinture murale, entre héritage et renouveau

- Esprit communautaire et art médiéval

- Bissière et l'Académie Ranson

- Le groupe Témoignage

Participation à des événements collectifs majeurs

Restaurer la primauté du spirituel

A. Gleizes et Moly-Sabata, une résidence d'artistes proche de Témoignage

- Les Jeunes Peintres de tradition française ou l'art sous l'Occupation

- Arts romans, art moderne et conversion : le cas du peintre Manessier

- Vitrail contemporain et architecture romane

- Un monde laïc à la recherche du sacré

Liste des illustrations

Bibliographie

Suite aux avant-gardes majeures du début du $\mathrm{XX}^{\mathrm{e}}$ siècle (cubisme, fauvisme, surréalisme), la nouvelle génération d'artistes qui émergea à l'approche de la Seconde Guerre mondiale en France (Jean Bazaine, Jean Bertholle, Roger Bissière, Elvire Jan, Jean Le Moal, Alfred Manessier, 
Edouard Pignon, Maria Helena Viera da Silva.... ${ }^{1}$ savait qu'elle ne pouvait se contenter d'imiter la peinture des aînés, fut-elle innovante. Emboîtant le pas des maîtres de l'art moderne, il leur fallait s'aventurer sur des voies inédites, conformes aux aspirations de leur temps. D'autant que leurs ambitions personnelles se rejoignaient dans une volonté commune de ne pas dissocier nouveauté des formes et contenu philosophique, spirituel ou religieux, pour répondre aux attentes d'un public en quête de sens dans une période troublée ${ }^{2}$. Or à ce titre, le cas de l'art roman se révéla pour eux un modèle du genre, tant sur le plan formel que par l'esprit.

\section{Recherche d'une voie propre}

Dans la fougue de sa jeunesse, cette génération se sent l'âme d'un médiateur entre l'art de son temps et le public, un peu déconcerté par ces formes inédites et ces couleurs franches. Le divorce entre le public et l'art moderne pouvait sembler consommé. Pour ces peintres, la quête d'un art qui véhicule un sens pour l'homme moderne passe par la rencontre de figures charismatiques et par l'adhésion à des groupes d'artistes et de penseurs qui se révèleront déterminants. Parmi eux, l'Académie Ranson avec Roger Bissière, le groupe Témoignage autour de Marcel Michaud et les Jeunes Peintres de tradition française avec Bazaine.

\section{Le rôle de l'art roman}

Le cours de fresque animé par Bissière à l'Académie Ranson dans les années trente s'inscrit dans un contexte de redécouverte de l'époque médiévale où l'art naissait d'un esprit religieux. Cours et publications se multiplient sur cette question. Beaucoup de jeunes artistes trouvent d'étonnantes similitudes entre l'art roman et leurs propres préoccupations formelles héritées du cubisme (abandon de la perspective linéaire, renoncement au modelé et au volume, simplification des motifs). Ce fut pour eux une révélation tant d'un point de vue artistique que spirituel. C'est pourquoi nombre d'historiens de l'art ont noté le retour à la simplicité du

\footnotetext{
${ }^{1}$ Nouvelle génération baptisée plus tard « La Seconde (ou Nouvelle) École de Paris ».

2 «Si l'exposition de 1938 [à la Galerie Breteau à Paris] eut tant de retentissement, c'est justement parce qu'elle allait bien au-delà de l'invention formelle, elle était en quelque sorte le témoin de l'inquiétude d'une époque. Pour s'étourdir, Paris avait beau multiplier les fêtes, les bals masqués, il n'en restait pas moins vrai que l'Allemagne s'armait, grondait, qu'Hitler hurlait, gesticulait, éructait et allumait des feux sur les places publiques, brûlant livres et peintures. Paris se cachait les yeux et pourtant là-bas, on assassinait les communistes et les juifs, les artistes fuyaient, la terreur était permanente» (dans Philippe Le Burgue, 2005, Bertholle, Neuchâtel, éd. Ides et Calendes, p. 25-26).
} 
Moyen Âge et la redécouverte des arts primitifs comme l'une des grandes conquêtes de l'art contemporain. Pierre Francastel raconte :

«L'une des sources d'idées les plus fécondes pour eux c'est la peinture romane. Ils sont férus de ces œuvres nouvellement étudiées où ils voient, d'une part des recherches d'invention pure, et d'autre part, les débuts de la tradition française (...). Les jeunes peintres contemporains considèrent que l'art roman fournit la preuve qu'il peut exister un art à la fois expressif et abstrait non soumis aux lois de la perspective linéaire $»^{3}$

Ce qui est déterminant, comme l'écrit encore Francastel, ce sont «certaines affinités sentimentales (...) qui se constituent à travers les âges, les familles d'artistes ». Pensée confirmée par les artistes, dont Manessier dans une interview : «On a une famille qui remonte dans la nuit des temps. On se sent très, très proches des Grecs, de l'art roman, de certains peintres, on peut les nommer au cours des siècles. Il y a des vérités qui sont des vérités collectives dans la peinture ». ${ }^{4}$

L'enseignement universitaire de Henri Focillon eut un grand impact sur les artistes (dont Bazaine qui suivit ses cours). Dès 1919, ce médiéviste en appelait à un retour aux origines :

«Revenons à nous-mêmes, à notre passé, à nos origines les plus lointaines, à tous les monuments de notre effort, à tout ce que nous avons donné d'intelligence et de vertu. L'histoire n'est pas un médicament aride, un recul dans le temps. C'est la mémoire des peuples. Elle ne se détourne pas, elle exhorte, elle entraîne à l'action $\gg .{ }^{5}$

L'une des idées forces de son ouvrage Vie des formes (1934) justifie cette exhortation. Selon lui, le temps n'est pas linéaire, sa durée est ponctuée d'avancées mais aussi de retours en arrière, de ruptures, de stagnations, de reprises du passé, suivant une structure cyclique. Il pose la notion de «famille spirituelle» sous laquelle se regroupent des artistes de différentes époques unis par une sensibilité commune, par exemple ceux de l'époque romane et ceux du début du $\mathrm{XX}^{\mathrm{e}}$ siècle, sachant que cet historien de l'art est un fin connaisseur des deux époques.

\footnotetext{
${ }^{3}$ P. Francastel, Nouveau dessin, nouvelle peinture, l'École de Paris, Paris, Librairie de Médicis, 1946, p. 167.

${ }^{4}$ Dans le film Les Offrandes d'Alfred Manessier de Gérard Raynal; couleurs, $52 \mathrm{mn}$, production Soleluna Films, CNAP et FR3, « Océaniques », 1992.

${ }_{5}^{5}$ Déclaration d'Henri Focillon le 23 mai 1918, dans Les Évocations françaises : les pierres de France, Paris, 1919, introduction, p. II (cité dans L. Bertrand Dorléac, L'Art de la défaite, 1940-1944, Paris, Seuil, 1993, p. 210).
} 

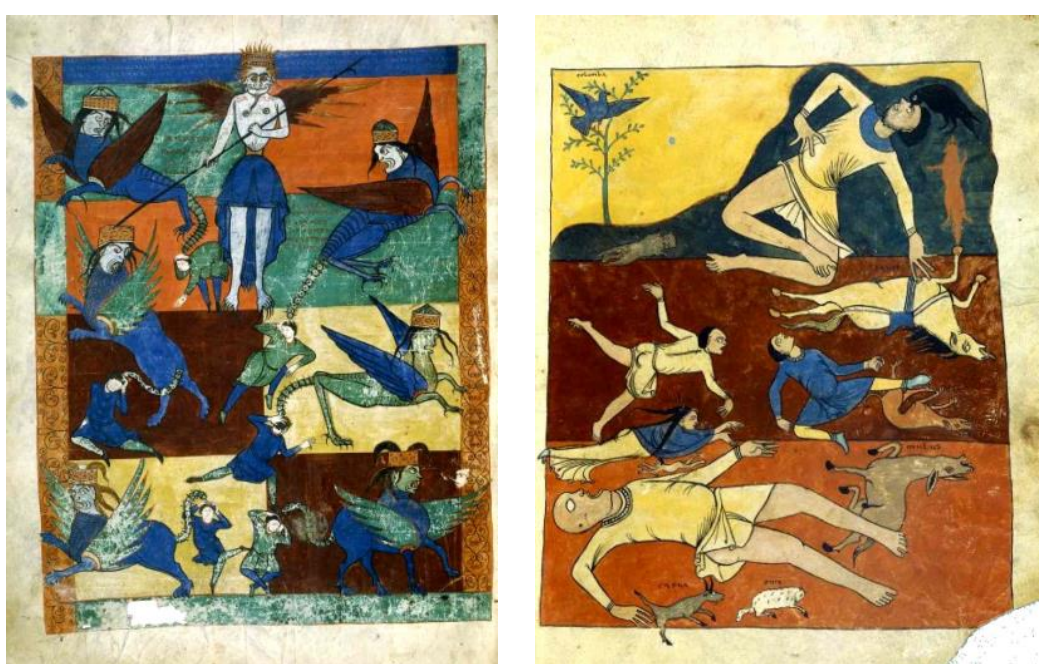

ill. 1, 2 Stephanus Garsia et deux anonymes, Apocalypse de Saint Sever (ou Le Beatus de Saint Sever), mi-XI siècle

Au registre des publications, on note l'importance de l'ouvrage Les Arts primitifs français édité en 1942 par Léon Gischia et Lucien Mazenod, comprenant de magnifiques reproductions. Les revues Minotaure puis Verve ${ }^{6}$ participent dans les années 1930-1940 à la diffusion de modèles artistiques variés à travers de nombreuses illustrations ${ }^{7}$ sur les arts primitifs, les cultures anciennes et l'art roman. La vue de quelques miniatures de l'Apocalypse de Saint-Sever $\left(\mathrm{mi}^{-} \mathrm{XI}^{\mathrm{e}}\right.$ siècle, ill. 1, 2) ${ }^{8}$, éditées en fac-similé en $1947 \mathrm{chez}$ Tériade, jouèrent pour nombre d'artistes un rôle décisif, avec leur figuration simplifiée et souvent brutale, leur chromatisme vif. ${ }^{9}$

La revue Art sacré $e^{10}$ consacra en 1945 un de ses numéros aux peintures romanes en France. Un parallèle y est souvent établi entre le style de Georges Rouault ${ }^{11}$, aux grands aplats colorés et aux cernes noirs, et les

\footnotetext{
${ }^{6}$ Minotaure, revue d'inspiration surréaliste éditée de 1933 à 1939 à Paris sous l'impulsion des éditeurs Skira et Tériade ; Verve, revue artistique et littéraire fondée à Paris par Tériade (38 numéros entre 1937 et 1960).

${ }^{7}$ Manessier se souvient : «Il y avait deux revues, Verve et Minotaure, qui étaient des revues essentielles (...). Un très beau numéro de Verve était consacré aux fresques de Saint-Savin et aux manuscrits du XII ${ }^{\mathrm{e}}$ siècle, avec un texte d'Aragon, je crois » (extrait de l'entretien de Claire Stoullig avec Manessier, publié dans cat. expo. Manessier, Grand Palais, Paris, éd. Skira, CNAP, 1993, p. 133-134).

${ }^{8}$ Ce manuscrit enluminé sur parchemin est un commentaire illustré de l'Apocalypse de Beatus de Liébana, écrit au VII ${ }^{\mathrm{e}}$ siècle, réalisé sans doute à l'abbaye de Saint-Sever.

9 Dans l'article «une tradition à haut risque» de Guitemie Maldonado, hors série Connaissance des arts sur l'exposition « L'art en guerre 1938-1947 », n555, MAM de la ville de Paris, 2012, p. 47.

${ }^{10}$ Publiée de 1935 à 1969 au service du renouveau de l'art sacré en France.

${ }^{11}$ Georges Rouault (1871-1958) appartient à la génération précédente.
} 
œuvres d'art roman, d'autant plus qu'il est considéré comme l'un des peintres religieux les plus importants du $\mathrm{XX}^{\mathrm{e}}$ siècle.

Les cahiers de Zodiaque contribuèrent eux aussi à diffuser des reproductions de l'art roman. Créée en mars 1951 par les Bénédictins de l'abbaye Sainte-Marie de la Pierre-qui-Vire (Yonne, France), en particulier Dom Angelico Surchamp ${ }^{12}$ et Dom Claude Jean-Nesmy, ce titre d'édition fut considéré comme une référence pour l'art sacré. Son premier numéro s'ouvrait sur un plaidoyer pour l'art sacré abstrait. Un an après sa création, Zodiaque consacra un numéro entier (71 pages) au peintre Albert Gleizes, avec de nombreuses reproductions et citations. ${ }^{13}$ Gleizes fit de nouveau l'objet de deux articles dans le numéro $25 .{ }^{14} \mathrm{Ce}$ peintre a aussi écrit dans les pages de la revue. ${ }^{15}$

\section{Albert Gleizes et un cubisme « à la romane »}

Pour Gleizes, il était inconcevable de faire de l'art sacré sans se référer à un «principe traditionnel», au risque sinon de produire un art d' « individualisme» (celui de l'artiste). Il reconnaissait la nécessité pour l'artiste de se «religare, relier» à une source commune. Conviction hautement partagée par Surchamp, pour qui ce peintre joua un rôle essentiel : "Grâce à Gleizes, j'ai compris la parenté qui relie l'art roman aux arts primitifs et à l'art moderne. Il me fit comprendre (...) surtout à quel point les recherches de l'art moderne - celui issu de Cézanne, Van Gogh et Gauguin rejoignaient formellement celles des $\mathrm{XI}^{\mathrm{e}}$ et $\mathrm{XII}{ }^{\mathrm{e}}$ siècles $» .{ }^{16}$

L'époque de référence pour un nouvel art sacré est le Moyen Âge selon Gleizes, «expression la plus exacte et la plus belle ». Nombreux tableaux témoignent de cette inspiration médiévale, comme Crucifixion (1935, ill. 3) Autorité spirituelle et pouvoir temporel (1939-40, ill. 4) ou Les Evangiles (1939-1941, ill. 5). Il va jusqu'à dire que l'artiste est « le serviteur de l'œuvre dont les structures vivantes devraient lui être fournies par les théologiens et les métaphysiciens », évinçant la possibilité pour l'artiste de pratiquer un pur jeu de formes et de couleurs sans âme.

\footnotetext{
${ }^{12}$ Dom Angelico Surchamp (1924-2018), peintre et fresquiste, médiéviste spécialiste de l'art roman.

${ }^{13}$ Zodiaque ${ }^{\circ}{ }^{6}$-7, janvier 1952, préface de Jean Cassou.

14 Dom Angelico Surchamp, "Albert Gleizes », et Thomas Greenwood, «L'esthétique religieuse du peintre Gleizes », Zodiaque, ${ }^{\circ} 25$, avril 1955, p. 20-55 et 56-58.

${ }^{15}$ Albert Gleizes, «Anne G. Dangar », Zodiaque, n²5, avril 1955, p. 8-9.

${ }^{16}$ Dans dossier «L'art roman au XX ${ }^{\mathrm{e}}$ siècle », revue Arts sacrés, 2009, p. 36.
} 

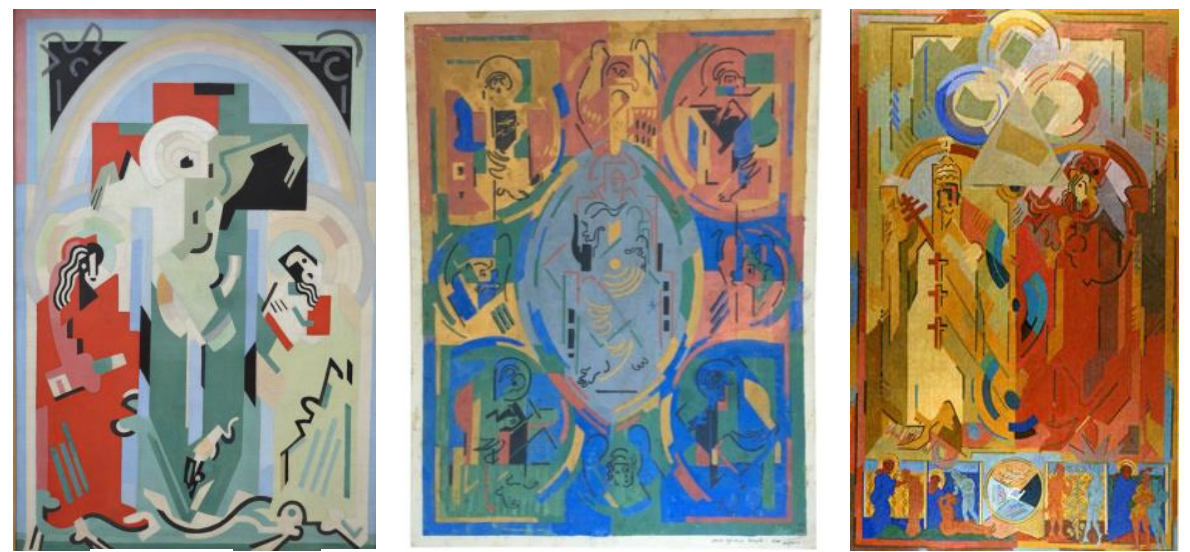

ill. 3, 4, 5 Albert Gleizes, Crucifixion, 1935 ; Les Evangiles, 1939-1941 ; Autorité spirituelle et pouvoir temporel, 1939-1940

Le Père Régamey (historien d'art et auteur, l'un des piliers de la revue l'Art sacré) n'a pas toujours été tendre à l'égard de la revue concurrente, Zodiaque. Sur une note concernant les revues d'art sacré, il en parle comme d'une «moinerie néo-romano-cubiste ${ }^{17}$, reprochant la sécheresse et le systématisme d'un excès de symbolisme (inspiré notamment de l'art roman).

En réalité, Régamey reproche à Gleizes d'appliquer des « recettes » toutes faites, cuisinées 《à l'antique » ou «à la médiévale» (comme l'utilisation systématique de nimbes, mandorles, arcs, etc. pour donner un côté glorieux aux personnages saints). Ce symbolisme nécessitait selon lui une initiation du grand public sous peine de rester une langue morte.

En mars 1945, dans ses notes pour «le cours Saint-Jacques » sur «Les tendances actuelles [de l'art religieux] ${ }^{18}$, Régamey considère que « [le cubisme] se prête peu à l'expression des réalités chrétiennes, à cause précisément de ce caractère abstrait, qui peut satisfaire l'esprit par sa rigueur, satisfaire les yeux par le charme des agencements de surfaces colorées, mais ne parle guère au cœur. Ainsi Albert Gleizes (...). Restera toujours un art pour initiés » (p. 10) ${ }^{19}$. Même si Régamey n'aime pas la manière dont le cubisme fait de l'art religieux, il reconnaît que Gleizes a sa place dans les expositions d'art sacré en France comme représentant d'une «tendance

\footnotetext{
${ }^{17}$ Feuillet daté du 20 avril 1953 dans Fonds Régamey, APDF (boîte 842-700.32).

${ }^{18}$ Notes du 10 mars 1945 dans Fonds Régamey, APDF (boîte Art sacré I - V-842-700.31).

${ }^{19}$ De même, Régamey pense que le surréalisme n'est pas adapté à l'art religieux, mais pour des raisons différentes : «Du surréalisme (...) dans l'art religieux, il ne peut être (tel du moins qu'il s'est manifesté jusqu'ici) que scandaleux ou risible» (notes pour «le cours SaintJacques » du 10 mars 1945 sur «Les tendances actuelles [de l'art religieux] », p. 13, Fonds Régamey, APDF, boîte Art sacré I - V-842-700.31).
} 
actuelle ». ${ }^{20}$

Ainsi, une littérature abondante sur l'art roman était diffusée dans les années 1940 en France, comme le rappelle Laurence Bertrand Dorléac dans L'Art de la défaite (1993). Un mémoire fut aussi consacré en 1994 à la revue Zodiaque (1951-1971): de l'art roman à l'art abstrait, un art sacré pour notre temps ${ }^{21}$, résumant en son titre l'affinité entre l'art roman et les nouvelles tendances picturales au $\mathrm{XX}^{\mathrm{e}}$ siècle. Et surtout, le rôle de Zodiaque pour nourrir le public à l'époque.

\section{L'art roman s'expose}

Dans les années trente, la culture médiévale s'enrichit également de belles expositions, comme celle sur Les Enlumineurs français du VIII au XV siècle à la Bibliothèque nationale en 1937, ou celle sur les œuvres d'art catalan du $\mathrm{X}^{\mathrm{e}}$ au $\mathrm{XV}^{\mathrm{e}}$ réunies au musée national du Jeu de Paume la même année, qui attiraient de très nombreux visiteurs.

L'enthousiasme des jeunes peintres fut aussi suscité par l'ouverture en $1937^{22}$ de nouvelles salles d'exposition consacrées à l'art roman, dont la surface fut triplée, ce qui permit à un large public d'accéder plus facilement aux trésors de l'art médiéval. Ces salles étaient le résultat d'un formidable travail de reproduction engagé par Prosper Mérimée ${ }^{23}$ au XIX ${ }^{\mathrm{e}}$ siècle. $^{24}$ «Quand on a inauguré le département des fresques romanes au musée des monuments français de Chaillot, les jeunes peintres de l'époque - Manessier, Estève...- ont été stupéfaits. Ils ont dit : 'C'est exactement ce qu'on cherche à faire !" ». ${ }^{25}$ Dans une lettre adressée à son épouse, datée de septembre 1945 et écrite suite à sa visite dans ce musée, Bissière exprime son enthousiasme pour les copies des portails de Moissac, Vézelay et Autun. Le sud-ouest de la France représente également pour lui une source d'inspiration extrêmement

\footnotetext{
${ }^{20}$ En 1947 (année des expositions d'art religieux contemporain à Nantes, Bordeaux, Lyon et Dijon), il avoue à René Déroudille : «Je crains de [de]voir faire une trop grande place à Gleizes qui mérite effectivement une place, mais qui ne doit pas être mis excessivement en vedette ; ce serait un très grave contresens » (Fonds Régamey, APDF, boîte Art sacré III - V842-700-33, pochette « Lyon 1947 »).

${ }^{21}$ Département Histoire, université Lyon Lumière, France.

${ }^{22}$ Lors de l'Exposition Internationale à Paris.

${ }^{23}$ À la fois écrivain, traducteur, critique, historien et archéologue, P. Mérimée (1803-1970) est connu comme une référence majeure en matière de protection du patrimoine. Il fut aussi Inspecteur général des monuments historiques.

24 «Depuis un siècle, la Direction générale des beaux-arts avait fait prendre à l'aquarelle des relevés à petite échelle des fresques qui décoraient les murs de plusieurs centaines d'églises médiévales françaises. Les œuvres avaient été reproduites sur place en grandeur réelle sur des toiles revêtues d'un enduit spécial qui leur donnait l'aspect de la pierre » (L'art de la défaite, 1993, p. 249).

${ }^{25}$ Dans interview de Dom Surchamp, «Le roman de Zodiaque », revue Arts sacrés, n², nov.déc. 2009 , p. 36.
} 
riche avec Souillac, Rocamadour, Toulouse et la cathédrale de Cahors. L'impact sur Bertholle de la découverte physique de l'art roman se reconnaît dans plusieurs textes et images consignés dans son Livre d'étude (19361942). ${ }^{26}$

De même, la pureté abstraite des abbayes cisterciennes (comme Notre-Dame de Sénanque ou le Thoronet, datées du XII ${ }^{\mathrm{e}}$ siècle) fascinait les jeunes artistes et était pour eux source d'inspiration spirituelle et formelle (beauté des lignes pures). En dressant un inventaire des nouvelles tendances artistiques depuis vingt ans, Georges Mercier évoquait les utilisations de l'esthétique non-figurative au service du christianisme dans l'art roman. Ce qui peut expliquer la référence à cet art du passé par certains peintres nonfiguratifs de la Seconde École de Paris qui se sont reconnus dans ces formes dépouillées et les ont pratiquées comme un « retour aux sources ». ${ }^{27}$

Un point mérite d'être fait ici sur la différence entre « art abstrait » et «art non-figuratif», puisque plusieurs artistes de la nouvelle génération furent classés plutôt dans la seconde catégorie. Contrairement à l'art abstrait, l'art non figuratif conserve un lien avec la réalité sensible mais ce lien dépasse la figuration classique, narrative, illusionniste, naturaliste. Manessier et Bertholle préféraient parler de «figuration allusive» que de nonfiguration, la négation induisant, à tort, toute absence de lien au réel. Pour l'art roman, on pourrait parler de «figuration symbolique » plus que de «figuration allusive »; dans les deux cas, le lien avec le réel est gardé sauf mais n'implique pas une imitation du réel.

\section{La peinture murale, entre héritage et renouveau}

L'époque romane est le temps de la grande expansion de la peinture murale, où des églises furent entièrement couvertes de peintures. A l'époque gothique, cet art cède la place au vitrail. En 1937, le musée des Monuments français à Paris se vit confier la mission de sauvegarder le souvenir des fresques romanes, délicates et fragiles, vouées à la destruction du temps (« les teintes s'atténuent, l'enduit peint s'écaille, se boursoufle et des morceaux de la précieuse pellicule de couleur tombent sur le sol. Et le mal est presque sans

\footnotetext{
${ }^{26}$ Dans ce document de travail, qui révèle ses sources de réflexion picturale autant que ses nourritures spirituelles et intellectuelles, sont collées des photos d'églises médiévales (intérieur et extérieur : un tympan, un bas-côté, trois colonnes avec chapiteau sculpté, p. 114, 140, 146, 147). Est aussi recopié un texte d'Elie Lebasquais sur le «Symbolisme du Christ en gloire aux tympans des églises médiévales », sans doute extrait de la revue Le Voile d'Isis, ${ }^{\circ}{ }^{184}$, avril 1935 (p . 7, 8, 13, 14 du Livre d'étude).

${ }^{27}$ Dans «L'Art abstrait dans l'art sacré. La tendance non-figurative dans l'art sacré chrétien contemporain », 1964, Paris, éd. de Boccard.
} 
remède $\gg^{28}$ ) en exécutant des copies aussi fidèles que possible. Parmi elles, les peintures de la voûte de Saint-Savin-sur-Gartempe (Vienne), qui incitèrent l'historien d'art Henri Focillon à qualifier cet ensemble de " Sixtine romane » et à lui consacrer vingt pages de texte (soit un tiers du volume) et quarante planches d'illustrations dans son ouvrage Les peintures romanes des églises de France paru en $1938 .{ }^{29}$ La partie romane du musée de la fresque fut inaugurée en 1945 et créa ainsi un nouveau département au musée des Monuments français.

Certes depuis Prosper Mérimée, le service des Monuments historiques avait fait exécuter des copies de fresques, mais cette collection extrêmement précieuse était réalisée à l'aquarelle sur papier, à échelle réduite et sur une surface plane. Les nouvelles répliques apparaissaient, quant à elles, rigoureusement conformes aux originaux «en l'état» (tons affaiblis, lacunes et blessures), en respectant les dimensions et le cadre architectural.

$\mathrm{Au}$ moment où «Vingt jeunes peintres de tradition française » exposaient leurs œuvres d'art moderne au nez et à la barbe de l'ennemi (en 1941 à la Galerie Braun à Paris, voir supra), la vingtaine de fresquistes poursuivait ce fastidieux labeur de copie fidèle, ainsi que le raconta Paul Deschamps : «L'Occupation n'arrêta nullement l'ardeur des artistes. On peut même dire qu'elle la stimula (...). Ils avaient le sentiment de faire œuvre patriotique (...) si bien qu'au lendemain de la Libération, les salles consacrées à la peinture romane étaient presque entièrement installées. Le 14 juin 1945 elles s'ouvraient au public ».

\section{Esprit communautaire et art médiéval}

Le retour de l'inspiration médiévale ne concernait pas uniquement les œuvres - fresque, sculpture, architecture, vitrail, tapisserie, enluminures mais aussi l'esprit qui animait les artistes et artisans à l'époque, et que l'Académie Ranson tendait à réveiller via sa dimension communautaire. «Les jeunes se plaignent de devoir travailler isolés, sans maîtres et sans compagnons. Aussi faut-il faire connaître l'effort libre et sincère entrepris par l'Académie Ranson (...) pour retrouver la forme des ateliers d'autrefois $\gg .^{30}$ Plus qu'un professeur, l'enseignant était un compagnon. D'autant que dans cette académie libre, les disciplines n'étaient pas cloisonnées. Il était possible d'assister à d'autres cours que celui auquel on était inscrit : peinture, dessin et croquis, gravure sur bois, arts décoratifs, sculpture et fresque (un art collectif

\footnotetext{
${ }^{28}$ Dans Paul Deschamps (1888-1974, conservateur puis directeur du musée des Monuments français), «Le département de la peinture murale au Musée des monuments français », Journal des savants, 1945, p. 84 www.persee.fr/doc/jds_0021-8103_1945_num_2_1_2754

${ }^{29}$ Paris, éd. Hartmann.

${ }^{30}$ Jacques Lassaigne, «Exposition de l’Académie Ranson », 7 juillet 1934, p. 10.
} 
par excellence proche de l'artisanat et de la notion du bel ouvrage). « Il faut que les élèves ne soient plus des élèves mais une corporation de véritables apprentis qui collaborent, se critiquent et se corrigent librement les uns les autres $\gg .31$

Ainsi de nombreux jeunes artistes des années 1930, notamment ceux qui fréquentaient l'Académie Ranson, se sont-ils ressourcés à l'esthétique romane dont ils allaient faire «un modèle et un stimulant» ${ }^{32}$ tant spirituellement que plastiquement. On peut parler d'imprégnation d'un état d'esprit car l'esprit médiéval offrait un modèle d'harmonie entre l'art et le public.

Cette étape fut capitale pour les jeunes artistes et cela à plusieurs titres : l'amitié durable avec leur aîné Bissière, les précieux échanges entre artistes, les expositions et chantiers en commun, la naissance du groupe Témoignage avec plusieurs élèves de cette académie. Au contact les uns avec les autres, ces peintres sont parvenus à une heureuse alliance entre la matière et l'esprit, telle qu'elle s'exprime dans la grande tradition de l'art français.

En 1965, soit trente ans après avoir fréquenté l'Académie Ranson, Bertholle se retrouve à diriger l'atelier d'art mural à l'École nationale supérieure des beaux-arts de Paris, nommé par André Malraux qui souhaitait dépoussiérer l'enseignement de cette institution. On ne peut qu'opérer un rapprochement entre la discipline qu'il enseigne - l'art mural - et l'existence du cours de fresque de Bissière qui l'aura sans doute sensibilisé à cet art collectif. Et au début des années 1980, Bertholle fonde sa propre école de peinture, l'Académie Saint-Roch. Son rôle auprès des élèves fut de même nature que celui de Bissière : guider, conseiller, éveiller.

\section{Bissière et l'Académie Ranson}

En 1923, Bissière est engagé à l'Académie Ranson pour animer un atelier de peinture et de croquis. Préparé par ses écrits sur la peinture murale, il ouvre un cours de fresque en novembre 1934, qu'il dirige jusqu'en 1939.

Située à Montparnasse, en plein centre artistique de Paris, 1'Académie Ranson est fondée en 1908 par les peintres nabis ${ }^{33}$ Paul-Elie Ranson (1861-1909) et Maurice Denis (1870-1943), ce qui lui assure dès le départ une réputation d'indépendance et un certain prestige.

\footnotetext{
${ }^{31}$ Propos de Harriet Cérésole rapporté par Pierre Courthion en 1933, Revue hebdomadaire (cité par A. Charvier dans «Une histoire, un lieu », cat. expo. Montparnasse années 30 (...) Éclosions à l'Académie Ranson, Rambouillet, Palais du roi de Rome, Belgique, éd. Snoeck, 2010, p. 28).

32 J. Bazaine, Le temps de la peinture 1938-1998, Paris, Flammarion, coll. Champ, 2002, p. 111.

${ }^{33}$ Né en marge de la peinture académique fin $\mathrm{XIX}^{\mathrm{e}}$-début $\mathrm{XX}^{\mathrm{e}}$, mouvement artistique postimpressionniste d'avant-garde.
} 
Dans cette longue période d'activité de 1908 à 1955, une décennie est particulièrement faste et féconde, celle des années trente. Autour des professeurs Bissière (pour la peinture) et Malfray (pour la sculpture), des dizaines de jeunes artistes, hommes et femmes de toutes nationalités se forment dans un esprit de liberté, d'échanges et d'amitiés durables, jusqu'à constituer le noyau du futur groupe Témoignage (dès 1936) et favoriser la renommée internationale de ces artistes vingt ans plus tard sous le nom de «Seconde École de Paris ». Plusieurs d'entre eux (Bissière, Manessier et Étienne-Martin) seront honorés à la Biennale de Venise, ou nommés professeur à l'École nationale supérieure des beaux-arts de Paris (Bertholle, Étienne-Martin, Wacker), preuve de la reconnaissance dont ils bénéficient dans les années 1960-1970.

Une des clés de l'épanouissement de ces jeunes artistes: l'enseignement prodigué à l'Académie Ranson s'exerce dans le respect de la diversité des personnalités et des inspirations. Ce lieu est voisin de nombreuses autres académies dites $«$ libres $»^{34}$, actives dans les années trente et souvent ouvertes à l'art moderne. Par exemple, les ateliers d'André Lhote et de Fernand Léger, où l'apprentissage du cubisme est largement diffusé, ou l'Académie de la Grande Chaumière. Elles incarnent un enseignement complet, indépendant, cosmopolite et de qualité qui favorise les rencontres et les échanges.
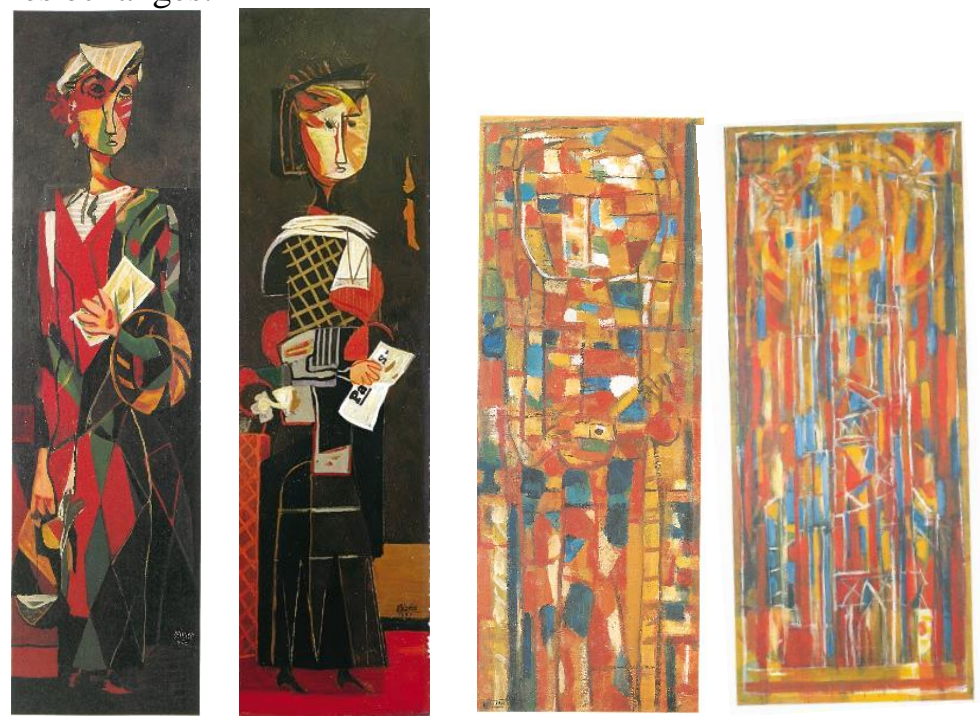

ill. 6, 7 Bissière, Figure debout, 1937 ; Grande Figure, 1937 ;

ill. 8, 9 Bissière, Joueuse de guitare, 1946-1947 ; Grande cathédrale, 1947

Avec sa Figure debout et sa Grande figure de 1937 (ill. 6, 7), Bissière montrait un exemple de fusion entre cubisme et art roman, avec des

\footnotetext{
${ }^{34}$ Par opposition à l'enseignement réputé « académique » de l'École des beaux-arts.
} 
formes simplifiées, expressives, dégagées de la perspective jusqu'à parvenir à un espace bidimensionnel. Ces recherches formelles, entre modernité et tradition française, se firent en commun sans que Bissière n'exerce plus d'influence sur ses élèves qu'eux en exerçaient sur lui. On note par exemple que les premiers tableaux de Bissière après la guerre (comme Joueuse de guitare ou Grande cathédrale datés de 1946-1947, ill. 8, 9) ont un point commun pictural avec ceux de Manessier quelques années plus tôt, comme Les Pèlerins d'Emmaüs (1943, ill. 14, infra), avec leur fragmentation colorée qui fusionne 1'espace et l'objet, mais associée aux couleurs vives de Matisse ou de Bonnard.

Proche des peintres cubistes André Lhote et Georges Braque, auteur de plusieurs articles sur le cubisme et de la première monographie sur Braque en 1920, Bissière discerne dans le cubisme un retour aux moyens traditionnels tels qu'on les trouve dans l'art roman ${ }^{35}$.

En 1939, Bissière quitte Paris et s'installe définitivement à Boissierette dans le Lot, en pleine campagne, avec pour seule compagnie son épouse, son fils et ses animaux : veaux, vaches, cochons... La radicalité de ce choix (dénuement, silence, dialogue avec la nature, émerveillement, méditation) explique la force et l'indépendance de son œuvre par la suite. Il recycle des matériaux très divers (vieux tissus, morceaux de bois usés, outils en fer, etc.), utilise des techniques picturales anciennes (peinture à l'œuf, à la fresque ou à la cire) appliquées sur des supports bruts (murs ou planche de bois non équarrie, tuile, cahier d'écolier, calendrier).

À Boissierette, il contemple la nature, vit au rythme des saisons, admire les variations de la lumière dans le paysage, s'émerveille des reflets du soleil sur le pelage des bêtes... Ce cadre, tel un retour aux «premiers âges du monde » selon ses propres termes, facilite l'éclosion d'une peinture nouvelle, plus spontanée et instinctive, comme une louange picturale de la nature.

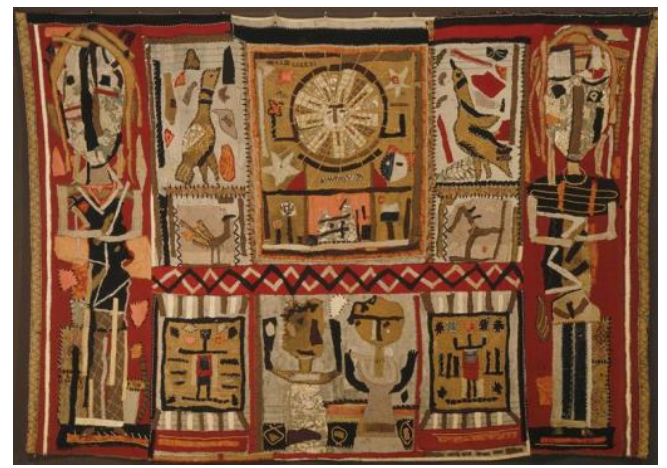

ill. 10 Roger Bissière, Soleil, 1945-1946

\footnotetext{
${ }^{35}$ C'est-à-dire simplification des formes, abandon de la perspective, du modelé, des effets de volume.
} 
Sa quête d'authenticité le conduit à abandonner le style figuratif au profit d'un art plus proche des arts primitifs, des dessins enfants ${ }^{36}$ ou de l'Art brut. ${ }^{37}$ Ses emprunts formels concernent aussi l'art mural du Moyen Âge : tapisserie, vitrail, sculpture et fresque. Dans les années 1945-1946, il conçoit une série de tapisseries (ill. 10), avec ce goût de l'artisan attaché au beau métier. ${ }^{38}$

Les titres de ses tableaux évoquent une inspiration pastorale et bucolique qui célèbre l'homme et son retour à l'état de nature. Une nature «que le peintre aime, qui le console, qui lui apporte, même, la joie. Et il ne cessera plus d'en prendre les Très Riches Heures, avec une fraîcheur, une intensité qui fait penser aux enluminures du Moyen Âge $\gg .{ }^{39}$ Son œuvre est la synthèse d'une multitude de sources d'inspiration :

«Je ne crois ni à l'expérience, ni à l'intelligence en matière de peinture, mais seulement à un instinct profond qui vient du fond des âges, à quelque chose de simple et de primitif qui est proche d'une gestation naturelle, je crois qu'on produit des formes et des couleurs comme un pommier produit des pommes, ce n'est pas un choix mais une fatalité ». ${ }^{40}$

Au final, cet homme humble et vrai, détaché de lui-même, a su produire une œuvre à son image: simplicité, poésie, innocence. Et transmettre aux artistes dont il était proche un supplément d'âme.

\footnotetext{
${ }^{36}$ «L'enfant sans effort va vers les synthèses et se fait une image du monde en retenant seulement les traits essentiels et caractéristiques » (dans L'Opinion, 20 mai 1916, cité dans Rose-Marie Arbour-Mayrand, Bissière et la tradition de la peinture, thèse de $3^{\mathrm{e}}$ cycle, Université Paris Sorbonne, 1973, René Jullian, directeur, p. 33).

${ }^{37}$ Terme inventé par Jean Dubuffet en 1945 pour désigner l'art de ceux qui n'ont ni culture ni formation artistique et dont la création est spontanée.

${ }^{38}$ Le beau métier consiste à bien connaître la nature du medium utilisé, ses possibilités et ses limites (d'après Bissière, L'Opinion, 15 sept. 1917). Mais d'après d'autres écrits (articles parus et textes dans son Journal manuscrit, rédigé entre 1908 et 1939), le beau métier n'a pas qu'une valeur technique ; il est porteur d'une garantie morale et artistique. Tel un rituel, il est investi d'un pouvoir allant au-delà des seuls gestes accomplis. "Au cours de la vie, on ne fait pas dans la peinture des découvertes techniques. On ne se perfectionne pas dans le sens matériel, mais seulement dans le sens spirituel. C'est soi-même qu'on apprend à connaître au-delà des aspects ordinaires (...) On ne prend chez les autres que ce qui nous appartient déjà » (Journal manuscrit, p. 28, cité dans Thèse Bissière, 1973, p. 90, op. cit. note 36 p. 8).

${ }^{39}$ Dans Jean-Clarence Lambert, «Hommage à Bissière », France Observateur, 9 avril 1959, $\mathrm{n}^{\circ} 466$, p. 20.

${ }^{40}$ Dans une lettre du 15 juillet 1951 au critique d'art Georges Boudaille.
} 


\section{Le groupe Témoignage}

Dans le sillage de l'Académie Ranson, les jeunes artistes connaissent différentes formes d'engagement dans les années 1930-1940, au service d'un art nouveau spirituellement nourri. Font partie de cette éclosion le groupe Témoignage (1936-1940) autour de la figure de Marcel Michaud, et l'exposition des Jeunes peintres de tradition française en 1941 avec le peintre Jean Bazaine.

Témoignage est un regroupement d'artistes, d'artisans et de penseurs autour de l'écrivain et galeriste Marcel Michaud à Lyon dans les années trente. Son ambition était de renouer avec le grand courant spiritualiste du Moyen Âge, de défendre des idées universelles sur l'homme, l'art, le monde, la spiritualité. Le groupe rayonnait au-delà de la sphère lyonnaise puisqu'il accueillait des artistes de diverses villes françaises ou même étrangères, et que l'un de ses objectifs était de sortir les villes de province de l'asphyxie culturelle et artistique (au début du siècle, seule Paris avait accès aux courants avant-gardistes). L'âme de Témoignage, selon Michaud : une «association par affinité d'inquiétude et non de certitude ${ }^{41}$, à laquelle opposer la vitalité de l'art et de l'artisanat, qu'il exposait dans ses galeries notamment.

L'art des peintres de Témoignage, dans son aspect formel, s'inscrit le plus souvent dans l'héritage cubiste pour les formes, fauve pour les couleurs et surréaliste pour l'atmosphère : des œuvres aux formes libres, dépouillées, géométriques qui n'imitent pas le réel, des aplats colorés dénués de volume, des couleurs souvent vives, avec une dominante de bleus et de rouges chez Le Moal et Bertholle, plus axées sur l'ocre, le vert et le brun chez Manessier.

Malgré de très fortes individualités, et l'absence d'une théorie esthétique commune, l'art de ces artistes présente donc une parenté formelle importante durant cette période, où apparaissent des motifs à caractère symbolique, allégorique et métaphorique, où le vocabulaire religieux se mêle aux symboles ésotériques (spirale, œil, astres...), caractéristique des artistes de Témoignage.

\section{Participation à des événements collectifs majeurs}

Dans ses articles et chroniques, Bissière prônait la nécessité de constituer des groupes d'artistes, d'architectes, d'artisans et de techniciens travaillant en commun et de façon anonyme. Ses idées correspondaient au contexte européen qui a vu naître le Bauhaus ${ }^{42}$ et d'autres groupes comme Moly-Sabata et Témoignage. Juste après l'ouverture de son cours de fresque,

\footnotetext{
${ }^{41}$ M. Michaud, «Pourquoi Témoignage », Le Poids du monde, n², 14 mars 1939, p. 45.

${ }^{42}$ Fondé à Weimar (Allemagne) en 1919 par Walter Gropius, le Bauhaus est un institut des arts et métiers surtout connu pour ses réalisations architecturales et pour son influence sur les arts plastiques. Kandinsky et Klee y ont enseigné plusieurs années.
} 
Bissière participait déjà avec ses élèves au Premier salon d'art mural de 1935, destiné à encourager les pratiques collectives.

Ces collaborations artistiques, dans le cadre de commandes de travaux d'architecture, sont l'occasion pour les jeunes artistes de pratiquer leur métier en lien avec le public. Ce qui répond à leur désir fondamental de réconcilier le public avec l'art de son époque, et d'associer art et artisanat, comme par le passé.

L'esprit du groupe Témoignage, qui souhaitait renouer avec la dimension communautaire de l'art roman, conduisit naturellement les artistes à collaborer à des chantiers. Plusieurs membres participent à des événements artistiques d'ampleur nationale et internationale. Les chantiers de 1937 et $1939^{43}$ font partie du « bilan » de Témoignage publié dans le numéro 3 de la revue du groupe, Le Poids du monde, en plus des expositions à Lyon, Grenoble et Paris de 1937 à 1939.

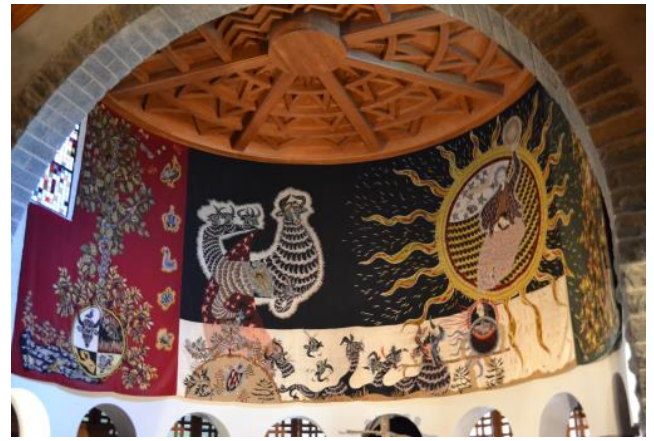

ill. 11 Jean Lurçat, $L$ 'Apocalypse, 1947
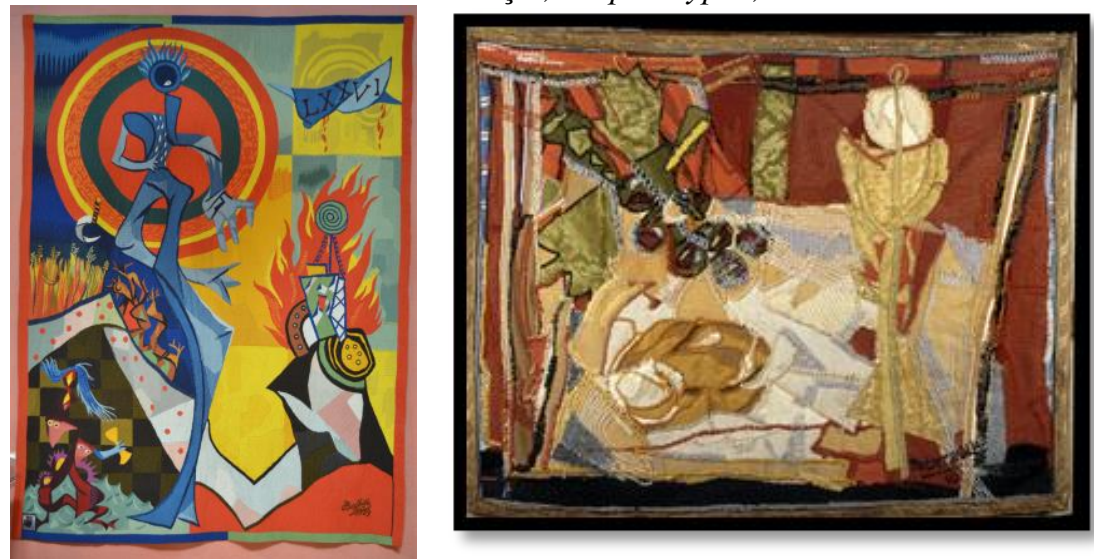

ill. 12, 13 Jean Bertholle, Glorification, 1946

et Devant d'autel (ou antependium), 1980

\footnotetext{
${ }^{43}$ En 1937, la décoration du pavillon des chemins de fer pour l'Exposition internationale des arts et techniques, événement majeur en France cette année-là. Et en 1939, réalisation d'un décor peint de $1500 \mathrm{~m}^{2}$ pour le plafond du pavillon français à l'Exposition universelle de New York.
} 
De ces expériences collectives avec l'Académie Ranson et le groupe Témoignage vient sans doute le goût du peintre Bertholle pour le monumental qui lui fit accepter, quelques années plus tard, des commandes de vitraux, mobilier, mosaique et. ${ }^{44}$ L'art de la tapisserie, qui connut ses heures de gloire au Moyen Âge, fut en quelque sorte réhabilité (avec Jean Lurçat surtout, et aussi Bissière et Bertholle, ill. 10 à 13). Dans l'éditorial d'une revue d'art, André Chastel écrivit ceci au sujet des tapisseries au $\mathrm{XX}^{\mathrm{e}}$ siècle: "L'action de Lurçat a été favorisée par le climat particulier de l'après-guerre, par le besoin d'un "retour aux sources", à une certaine pureté originelle. Mais elle ne sut pas éviter une contradiction de fait : la mise en œuvre de techniques perfectionnées à l'extrême pour parvenir à une apparence rudimentaire, artisanale, pour répondre à un primitivisme artificiellement provoqué $\gg .{ }^{45}$

\section{Restaurer la primauté du spirituel}

En plus de formes artistiques nouvelles, Témoignage prône la quête d'un renouveau spirituel, avec le souci de créer un style qui ait une âme. Pour cela, le groupe se tourne vers la tradition, en particulier l'art roman, témoin d'une époque où l'art était une chose publique, fédératrice, et non une production d'élites à destination d'autres élites - du moins dans sa vision fantasmée à l'époque. Selon Michaud, il faut quitter l'amour de l'esthétique comme fin en soi pour atteindre d'autres réalités où l'être humain retrouve une place centrale, pour redécouvrir effectivement l'homme perdu. ${ }^{46}$ Dans cette volonté d'un retour à l'esprit en art, une certaine filiation est aussi revendiquée avec la préhistoire.

En réalité, ce qui intéresse Témoignage au premier chef est l'Homme et sa place dans le monde, avec une grande place accordée à la spiritualité dans un sens très ouvert. Cherchant à créer une solidarité nouvelle, Témoignage établit des passerelles entre les disciplines, les religions, les cultures. «Comment? En retournant aux sources, en réinjectant à l'art la spiritualité perdue depuis que la Renaissance a remplacé le mythe de Dieu par celui de l'Homme $\gg^{47}$

\footnotetext{
${ }^{44}$ Le goût de Manessier pour le monumental lui vint plutôt de ses études à l'École des beauxarts de Paris d'abord en section architecture. Il honora de nombreuses commandes de vitraux de 1948 jusqu'en 1993, année de son décès.

${ }_{45}$ André Chastel, L'Histoire de l'art : fins et moyens. Éditoriaux de la "Revue de l'art" 19681978, Paris, Flammarion, coll. Champ esthétique, 1980, p. 253 (édito n²8, 1973).

${ }^{46}$ Jean Cehavalier, «La Renaissance de l'Homme », Le Poids du monde, n², 14 mars 1939, p. 77 (cité dans Laëtitia Blanchon-Gaudin, Regard sur Témoignage, mémoire de master I d'histoire de l'art, Lyon, Université Lumière Lyon 2, 2010, Annie Claustres, directrice, 2010, p. 21).

${ }^{47}$ Dans texte anonyme, «Le sentiment mystique dans l'art actuel », Connaissance des arts,
} 
La réflexion de Témoignage est alimentée par plusieurs artistes et penseurs, au gré des rencontres de l'un ou l'autre membre. Les philosophies orientales, la religion chrétienne et l'ésotérisme sont parmi les principales sources d'inspiration, à travers quelques figures marquantes gravitant autour d'eux.

Parmi ces figures marquantes, le peintre Louis Thomas, grand admirateur de l'écrivain catholique Léon Bloy (1846-1917). Ce dernier déplorait le déclin de la religion et l'indifférence envers elle. Selon Bloy, l'art et la culture pouvaient remédier à la déchéance spirituelle caractéristique de l'époque à condition de garder sauf un lien avec la transcendance, avec un principe supérieur et de réhabiliter certaines valeurs humaines. ${ }^{48}$

Les ouvrages de René Guénon ${ }^{49}$, que Michaud avait lus avec fascination, contribuèrent eux aussi à éveiller les consciences sur les besoins du monde moderne. Sur les conseils de Michaud, Bertholle, Étienne-Martin, Stahly et d'autres s'engagèrent dans la lecture de textes comme le Symbolisme de la Croix ou Aperçus sur l'Initiation. ${ }^{50}$

Dans Orient et Occident paru en 1924, Guénon insiste sur ce que le monde occidental a égaré depuis le Moyen Âge. Et dans La crise du monde moderne $^{51}$, il situe à la fin du Moyen Âge le commencement de ce qu'il appelle une décadence. Pour lui, l'humanisme est symptomatique, par l'abstraction qu'il fait des principes supérieurs en ramenant tout à l'être humain, d'un glissement sur la pente fatale de l'individualisme, cause de la déchéance au $\mathrm{XX}^{\mathrm{e}}$ siècle. Cependant, cette vision pessimiste chez Guénon est compensée par l'activité créatrice de Témoignage, qui contribue à réenchanter le monde à travers la production d'œuvres d'art et leur diffusion.

Ainsi la religion occupe-t-elle une place importante au sein de Témoignage, mais plutôt dans le sens de ce qui recueille les traditions et relie les hommes entre eux (conformément aux deux sens étymologiques du mot) que dans le sens d'une religion particulière, dogmatique et institutionnelle. Michaud ne se convertit d'ailleurs jamais malgré le prosélytisme de certains fervents catholiques autour de lui. Le sentiment religieux qui anime la plupart des membres de Témoignage ne concerne pas le seul christianisme mais aussi un sacré plus laïque. Autant de formes différentes au service d'une même ambition : réinjecter du spirituel dans l'art.

\footnotetext{
août 1960, p. 65 (cité dans catalogue exposition Paris-Paris : 1937-1957, éd. Centre Pompidou, 28 mai-2 nov. 1981, p. 90).

${ }^{48}$ Léon Bloy dans Jean-Luc Maxence, René Guénon, le philosophe invisible, Paris, Presses de la Renaissance, 2001, p. 140 (cité dans Témoignage Blanchon, 2010, p. 30-31).

${ }^{49}$ Une des figures les plus marquantes de l'occultisme français dans la première moitié du $\mathrm{XX}^{\mathrm{e}}$ siècle.

${ }^{50}$ Bertholle cite René Guénon à deux reprises dans son Livre d'étude 1936-1942, p. 13.

${ }^{51}$ René Guénon, La crise du monde moderne, Paris, Bossard, 1924 (rééd. 2009).
} 


\section{Albert Gleizes et Moly-Sabata, une résidence d'artistes proche de Témoignage}

En qualité de théoricien du cubisme ${ }^{52}$ et peintre d'un cubisme à caractère religieux, Albert Gleizes joua lui aussi le rôle d'inspirateur au sein de Témoignage. En 1921, Michaud publie un premier article consacré à ce peintre, qu'il rencontre en 1930 à Moly-Sabata. Dans ce lieu, Michaud écrivit plusieurs articles, dont un consacré précisément à cette expérience de retour à la terre. ${ }^{53}$ L'année suivante, il rend compte d'une conférence de Gleizes : « Artisanat et retour à la terre ». ${ }^{54}$

En créant Moly-Sabata, Gleizes avait mis en pratique une organisation collective de l'art, qu'il prônait contre l'individualisme. En ce lieu résonnaient ses idées majeures : la conviction d'une vérité absolue dans l'art, l'attrait de l'absolu dans sa simplicité dépouillée, le retour aux techniques premières, les fortes influences primitives, l'attrait de l'art populaire (par opposition à l'art de salon, sophistiqué, raffiné, mondain). La proximité avec la nature et l'artisanat maintenait les pensionnaires dans une position anticapitaliste. La pensée de Gleizes était considérée par Michaud comme «révolutionnaire comme fut révolutionnaire le christianisme sous la domination romaine ».

Indépendamment de sa réserve sur cette vie en autarcie, Michaud voyait en Gleizes l'un des premiers ayant compris la nécessité de discipliner le jeu des formes à une pensée supérieure ${ }^{55} \mathrm{Ce}$ que confirme J.-J. Lerrant, interviewé dans le film sur Témoignage : «Il y a aussi l'influence de Gleizes, post-cubiste, qui cherche un retour à la tradition, à travers l'art roman, les grands courants de l'art, l'éternité de l'art, et même une espèce d'art collectif $\gg{ }^{56}$

\footnotetext{
${ }^{52}$ Parmi ses écrits : Du cubisme avec Jean Metzinger (1912), avec cette idée « que le tableau n'imite rien »; Du cubisme et des moyens de le comprendre l'Occident chrétien (1930); Art et religion, art et science, art et production (1933); Homocentrisme ou le retour de l'Homme Chrétien (1937). Albert Gleizes a notamment exposé sa conception de la peinture moderne et approfondi la rupture avec les traditions nées de la Renaissance. Il leur reproche un asservissement au monde sensible qui se traduit pour le peintre par une dépendance excessive envers le sujet traité comme un spectacle.

${ }_{53}$ M. Michaud, «Un effort sympathique : Moly-Sabata », L'Effort, n' ${ }^{\circ} 325$ et 326, 17 et 24 septembre 1932, Fonds M. Michaud, MBA de Lyon. En 1947, Michaud écrit un autre article sur Gleizes : "Vers la signification ", Albert Gleizes. Le Cubisme et son dénouement dans la tradition, Lyon, Nouvel Art, 1947.

${ }^{54}$ Dans L'Effort, 23 décembre 1933.

${ }_{55}^{5}$ D'après M. Michaud, «Pourquoi Témoignage », Le Poids du monde, n², 1939.

${ }^{56}$ Dans Daniel Le Comte, Le groupe Témoignage, Accord CLC productions, 2000, couleurs, $52 \mathrm{mn}$.
} 


\section{Les Jeunes Peintres de tradition française ou l'art sous l'Occupation}

Les peintres non-figuratifs se connaissent presque tous dès 1935 et commencent à exposer ensemble. Certains fréquentent l'Académie Ranson, où professe Bissière, et plusieurs participent aux expositions du groupe Témoignage, autour de Michaud. Contrairement à ce que l'on pourrait imaginer, la peinture française continua à faire preuve d'une grande vitalité durant la Seconde Guerre: "Peindre pouvait signifier faire un acte de présence, exister sans renoncer, en un mot résister $\gg .{ }^{57}$

Le premier signe d'un réveil collectif après la terrible défaite de $1940^{58}$ fut l'exposition qui s'ouvrit le 10 mai 1941 à la Galerie Braun à Paris sous le titre Vingt jeunes ${ }^{59}$ peintres de tradition française ${ }^{60}$, à l'initiative du peintre Jean Bazaine et de l'éditeur André Lejard. Ce titre masque la réalité d'une peinture avant-gardiste, pour contourner l'interdiction d'exposer un art qualifié de dégénéré par les Nazis.

Considérée comme militante dans ce contexte, l'exposition présente une peinture non-figurative aux curiosités plastiques communes. Ces Jeunes peintres renouent autant avec une tradition ancienne (art roman) que moderne (Bonnard, Picasso, Braque et Matisse). Ils sont les acteurs d'une tradition vivante, se démarquant de l'académisme prôné par l'ennemi. Le succès de cette exposition prouva que l'esprit français avait survécu à la défaite militaire, que les Nazis n'étaient pas parvenus «à mettre complètement au pas le monde de la création, et ce malgré les interdictions d'exposer, les saisies et les spoliations $»{ }^{61}$

Comme Bissière et les artistes proches de l'Académie Ranson, Bazaine se tourna rapidement vers le passé comme source d'inspiration. En 1934, il écrit: "Nous pourrons tâcher, si nous savons rester modestes, de retrouver l'attitude spirituelle des hommes du Moyen Âge ». En 1942, faisant référence à un temps idéal et soulignant la religion comme élément moteur de la création, il dira «qu'il manque à notre époque l'équivalent de ce puissant ferment que fut au Moyen Âge la religion, là est le drame. Où trouver un

\footnotetext{
${ }^{57}$ Dans catalogue exposition « Bazaine 1941-1947 : les années décisives », Galeries nationales du Grand Palais, Paris, 30 mars-28 mai 1990, p. 139.

${ }^{58}$ «Il ne faudrait tout de même pas que notre défaite militaire fît admettre par extension une déroute générale de tout ce que notre civilisation a donné jusque-là de meilleur. C'est faire aux guerres la part trop belle que de leur supposer une telle influence sur les destinées de l'esprit (...). En réalité, guerres, révolutions, "catastrophes mondiales", tout cela ne change pas grandchose à la marche profonde de l'esprit. » (dans J. Bazaine, "Guerres et évasions », NRF, $\mathrm{n}^{\circ} 326,1^{\mathrm{er}}$ avril 1941, repris dans Le temps de la peinture, 2002, p. 20, op. cit note 32 p. 7).

${ }^{59}$ Ces artistes ont entre 30 à 45 ans.

${ }^{60}$ Il s'agit de Bazaine, Beaudin, Berçot, Bertholle, Bores, Coutaud, Desnoyer, Gischia, Lapicque, Lasne, Lautrec, Legueult, Le Moal, Manessier, Marchand, Pignon, Roger, Singier, Tal Coat et Walch.

${ }^{61}$ Dans Véronique Bouruet-Aubertot, «La vie artistique sous l'Occupation », hors-série Connaissance des arts sur «L'art en guerre 1938-1947 », n555, 2012, p. 28.
} 
instrument de coagulation, d'unité de l'esprit, aussi décisif? ». L'article « La jeune peinture expose ${ }^{62}$ expliquait :

«Ce n'est pas à une tradition prônée par Vichy que se réfèrent la plupart de ces jeunes peintres mais à celle, "authentique", de l'art médiéval et plus particulièrement de l'art roman. Rejet de la perspective linéaire héritée de la Renaissance, adoption des vitraux, émaux cloisonnés et tapisseries du Moyen Âge et aussi leçons tirées du fauvisme et du cubisme qui, bien que plus récents, font également partie intégrante de notre tradition picturale (...)».

En prolongement de cette exposition, Gaston Diehl présente en février 1943 Douze peintres d'aujourd'hui à la Galerie de France à Paris, légitimant ainsi le groupe des Jeunes peintres de tradition française. Leur travail est soutenu dans la presse et à la radio, des écrivains leur consacrent livres, préfaces et articles. Ces jeunes artistes unissent leur solitude en se retrouvant dans les arrière-salles des cafés de Montparnasse pour discuter peinture. Là se dessine un esprit commun.

Outre la ressemblance formelle de leurs toiles, ces artistes partagent certaines idées sur leur pratique artistique, notamment la volonté de se rapprocher du public et de revaloriser une pratique plus artisanale de l'art, c'est-à-dire plus humble et moins élitiste, toujours suivant le modèle de l'art roman.

$\mathrm{Si}$ dès les débuts de l'Occupation une véritable machine de propagande s'était mise en place pour contrôler, en l'épurant, la scène culturelle française, les Jeunes peintres allaient incarner à la Libération la relève française. Celle qui avait résisté à l'occupant par l'art, soutenue par quelques critiques, historiens de l'art et galeristes. ${ }^{63}$

\section{Arts romans (fresque, architecture, musique), art moderne et conversion : le cas du peintre Manessier}

Il n'est pas impossible que la sensibilité de Manessier à l'art roman depuis les années trente (bien éloigné du style saint Sulpicien dont il disait le plus grand $\mathrm{mal}^{64}$ ) ait constitué un terrain favorable à sa conversion religieuse

\footnotetext{
${ }^{62}$ Dans La NRF, août 1941.

${ }^{63}$ D'après L. Bertrand Dorléac, «L'art sous 1'Occupation », Encyclopaedia Universalis (internet 2012).

${ }^{64}$ Cette expression, à connotation péjorative, qualifie des objets religieux (souvent des sculptures) produits à l'échelle industrielle pour orner les églises. De qualité médiocre et d'un style très mièvre, ils ne reflétaient pas, selon les dires de beaucoup d'artistes, de croyants, de clercs et de religieux, la beauté de la foi chrétienne. Ce style sévit à partir de 1850 et conduira certains artistes et amateurs d'art à initier un mouvement de renouveau pour tenter de sortir
} 
en septembre 1943 à la Grande Trappes de Soligny (datée du XII ${ }^{\mathrm{e}}$ siècle). D'autant qu'écouter du chant grégorien ${ }^{65}$ dans une église romane peut apparaître comme un modèle d'authenticité artistique, culturelle et spirituelle, dans une forme $d^{\prime}$ '«art total» capable de provoquer une vive émotion religieuse.

Dans son tableau Salve Regina (1945, ill. 15), directement inspiré du cantique traditionnel entendu au moment de sa conversion, s'affirme l'intimité entre émotion religieuse et création artistique telle que Manessier l'avait découverte avec enthousiasme au sein de l'art roman.
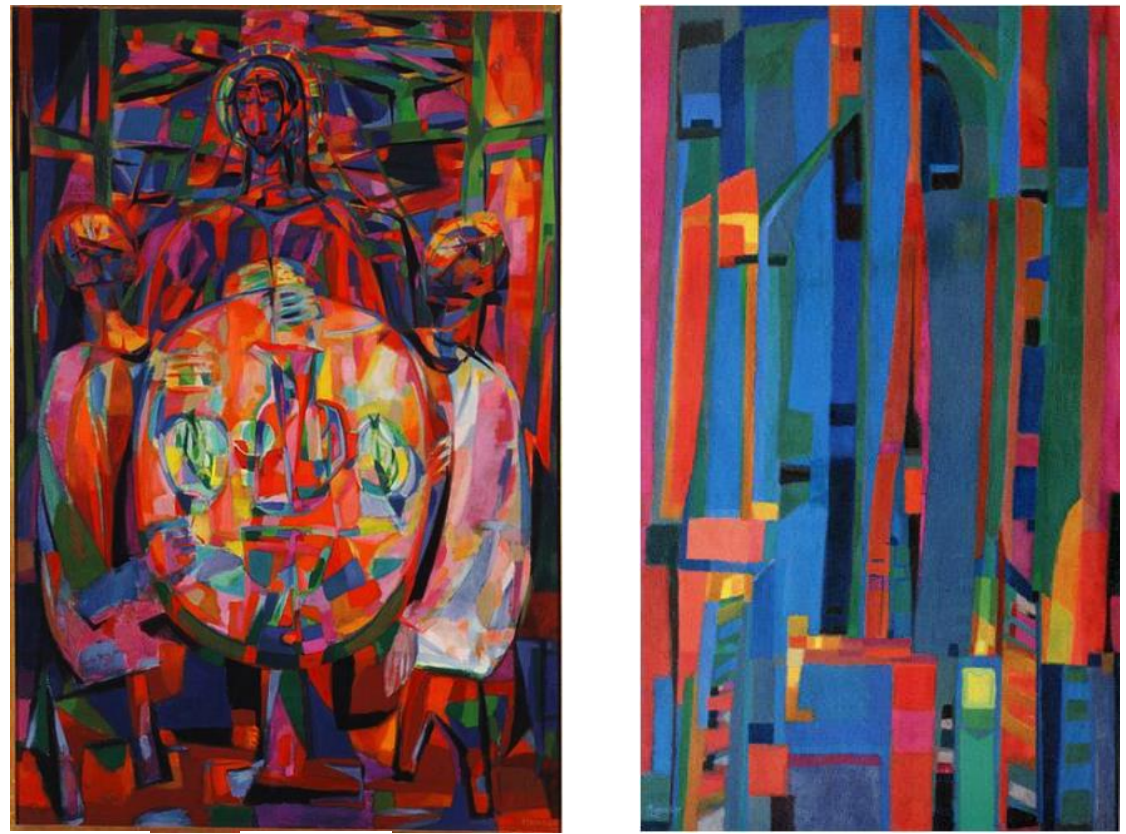

ill. 14, 15 Alfred Manessier, Les Pèlerins d'Emmaüs, 1943 ; Salve Regina, 1945

Le Salve Regina est directement inspiré du moment où Manessier reçut le don de la foi, en entendant le cantique chanté par les moines $a$ capella à la tombée du jour, dans la chapelle de l'abbaye. Au premier abord, il fait figure de tableau abstrait, composé essentiellement de formes verticales, dans une dominante colorée de bleus intenses et de roses très lumineux à la surface de la toile (aucune profondeur ni perspective). Ce

\footnotetext{
l'art sacré de cette impasse, dont les Pères dominicains Régamey et Couturier avec la revue l'Art sacré. «Saint-Sulpice, pour moi, c'est effroyable... (...). La vérité valait mieux que cela, elle valait infiniment mieux que son imagerie ; et je pense que c'est mal servir le peuple que de faire croire que sainte Thérèse, c'est ça! Si c'est ça, moi je rends mon billet, je m'en vais ailleurs ! » (dans interview de Manessier sur l'art sacré par Pierre Cabanne et d'autres, $34 \mathrm{mn}$, production France Culture, diffusée le 20 avril 1973).

${ }^{65}$ Art musical qui connut un grand essor, et son âge d'or, à l'époque romane grâce à la lente introduction au XIe siècle de la portée avec des lignes.
} 
tableau synthétise toutes les caractéristiques du style non-figuratif en maintenant la réalité d'un moment vécu sous des formes stylisées et symboliques, ouvrant un accès au visible (formes et couleurs) en passant par l'invisible d'une émotion musicale et religieuse, via le medium peinture. On notera par exemple le symbolisme de la couleur bleue, qui évoque le vêtement de la Vierge, et les verticales qui rappellent à la fois l'élévation du lieu, l'orgue et l'élévation spirituelle.

Le dépouillement musical des chants grégoriens est comparable à la non-figuration en peinture, et à la poésie en matière de création littéraire. Cette toile est caractéristique du basculement de Manessier vers la nonfiguration dans les années 1940. Car au moment où il décide d'abandonner la figuration, il sent comment «la musicalité de la peinture est à même d'exprimer les choses invisibles ». ${ }^{66}$ "Mon Salve Regina c'est une impression de la Trappe ${ }^{67}$, disait Manessier.

Ainsi la question religieuse se confond-elle toujours avec celle de l'art chez ce peintre. Seule la beauté sacrée de l'art roman, et les formes nouvelles d'art sacré proches de l'abstraction, pouvaient trouver grâce à ses yeux et constituer un terrain favorable pour une conversion. L'idée d'un art qui servirait, ou desservirait, la foi apparaît ici en filigrane : l'art roman, par son dépouillement symbolique et sa beauté pure, pouvait servir la foi chrétienne là où la figuration extrême (comme le style saint-sulpicien) pouvait éloigner de l'Eglise.

Quant au tableau les Pèlerins d'Emmaüs de 1943 (ill. 14) : la bidimensionnalité de l'espace pictural (que le cubisme et le fauvisme avaient réintroduite dans l'art en abolissant la perspective) et le recours aux cernes noirs pour encadrer les figures autour d'une table ovale relevée à la verticale sont autant d'éléments qui évoquent l'art médiéval. Au lendemain de sa conversion, Manessier aspirait à une peinture porteuse de l'innocence perdue des artistes :

« Si nous avions la pureté évangélique des Primitifs, si nous pouvions poser sur la nature un regard chargé de toute l'innocence de l'amour, alors peut-être nous pourrions peindre le sacré, comme ils le firent. Mais nous sommes des hommes de ce siècle : brisés, éclatés. Sur la toile surgit, que nous le voulions ou non, un mensonge qui est celui même de notre société.

En sortant de la Trappe, j'ai voulu exprimer ce que j'avais éprouvé grâce à trois figures sacrées autour d'une table. C'était faux, presque intolérable. J'ai donc pensé qu'en rejetant la figuration, j'exprimerais plus aisément ce que je ressentais $»{ }^{68}$

\footnotetext{
${ }^{66}$ Dans le film G. Raynal, 1992, op. cit. note 4 p. 3.

${ }^{67}$ Bernard Dorival, « Manessier, artisan religieux », revue $L^{\prime}$ Oeil, n ${ }^{\circ} 10$, oct. 1955, p. 28.

${ }^{68}$ Dans Jean Clay, « Manessier, ma vérité de peintre », Réalités, n²02, nov. 1962, p. 100.
} 
Deux ans plus tard, le Salve Regina confirmait la capacité de la nonfiguration à exprimer une émotion religieuse là où un sujet narratif (dans l'Evangile selon saint Luc, ch. 24, versets 13 à 35) avait failli, selon Manessier.

En réalité, les cubistes avaient déjà démontré l'importance d'un ressourcement aux arts primitifs, plus instinctifs, moins intellectuels que l'art de la Renaissance. Les propos de Manessier permettent une relecture du paysage artistique de son époque, pris entre le désir d'innover et la nostalgie d'une certaine tradition. Comme si Manessier avait fait entrer dans son atelier, au retour de la Trappe, le questionnement esthétique de son temps, ce monde lä̈que qui a besoin de retrouver le sens du sacré ${ }^{69}$. Et qu'il réaffirmait le rôle de l'artiste, se laisser inspirer :

«Un coin de ce fossé [entre l'art sacré et l'art] se trouvera comblé lorsqu'on aura reconnu à toute création artistique digne de ce nom qu'elle est marquée du sceau de l'Esprit. Une œuvre faite par un artiste, même complètement athée, relève du Don ; et procédant d'un travail intérieur est indélébilement marquée principalement dans les arts magiques (art nègre, Picasso, etc.) et c'est ce qu'avaient si bien senti les artistes romans en leur temps ${ }^{70}$

\section{Vitrail contemporain et architecture romane}

Sur les affinités artistiques entre passé et présent, on ne saurait oublier la manière dont le vitrail contemporain a prit place dans certains édifices anciens dont les verrières ont été détruites pendant les deux guerres mondiales. Manessier réalisa de nombreux ensembles de vitraux, certains dans des édifices anciens. Mais les vitraux de Soulages à l'abbatiale SainteFoy de Conques ${ }^{71}$ révèlent à eux seuls les affinités possibles entre abstraction et art roman (ill. 16), même si ce choix fut fortement décrié en raison du grand dépouillement des verrières, créant une atmosphère «froide » au goût de certains, surtout aux jours de mauvais temps...

\footnotetext{
${ }^{69}$ Ibid., p. 25.

${ }^{70}$ Propos de Manessier cités dans L'Art religieux actuel, cahiers de « Servir », Toulouse, 1948 (repris par C. Bourniquel et J. Guichard-Meili dans Les Créateurs et le sacré, 1956, p. 212).

${ }^{71}$ Sur Soulages, se reporter à l'article de V. Buisine dans Polar-isations francophones. Cinquante nuances de noir, actes du colloque international, journées de la francophonie, $\mathrm{XXII}^{\mathrm{e}}$ édition, Iasi, 24-25 mars 2017, Iasi (Roumanie), éd. Junimea, p. 231-238
} 


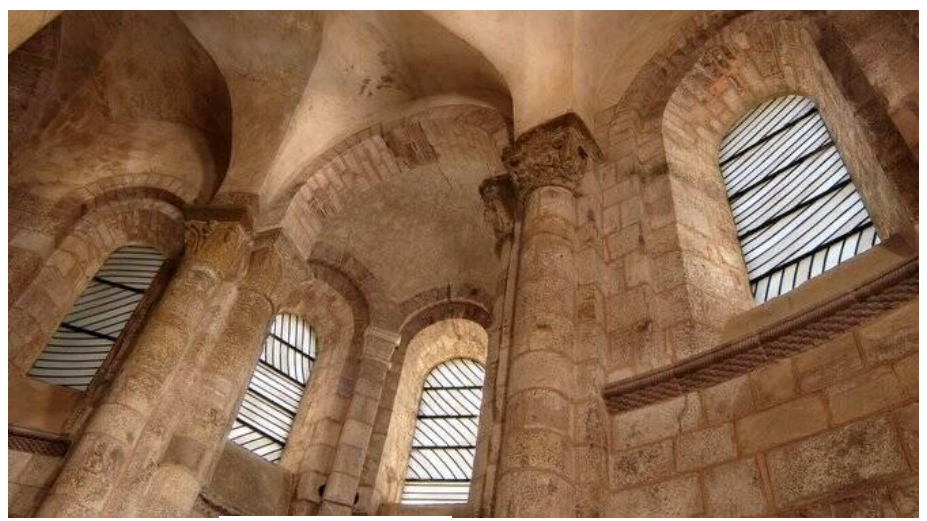

ill. 16 Soulages, vitraux à Conques

Né à Rodez en 1919, à une quarantaine de kilomètres de Conques (Aveyron, France), Soulages a confié sa fascination depuis son plus jeune âge pour l'art roman, les grottes préhistoriques, les vieilles pierres et les matériaux usés par le temps. Le seul projet de vitraux qu'il accepta fut celui de Conques, chef d'œuvre de l'architecture romane (XI ${ }^{\mathrm{e}}-\mathrm{XII}^{\mathrm{e}}$ siècles), classée au patrimoine mondial de l'UNESCO. Lieu où, raconte-t-il, est née sa vocation d'artiste ${ }^{72}$. Ses cent quatre verrières l'occupèrent huit ans (de 1986 à 1994) et nécessitèrent de nombreux essais techniques pour trouver la qualité de verre adéquate.

Par ailleurs, l'art de Soulages fut souvent l'occasion d'aborder la question de l'art moderne versus l'art du passé : en 1995, lors d'une conférence organisée par le Centre européen d'art et de civilisation médiévale de Conques sur le thème «De la pertinence de mettre une œuvre contemporaine dans un lieu chargé d'histoire », où il s'entretint avec Jacques Le Goff. Et en 2019 lorsque le philosophe Michaël de Saint Cheron convoqua le regard des médiévistes Georges Duby et Jacques Le Goff pour son livre Soulages. D'une rive à l'autre (éd. Actes Sud).

\section{Un monde laïc à la recherche du sacré}

Les jeunes peintres des années 1930-1940 ne furent pas les seuls à s'intéresser à l'art roman. Les "grands aînés » de l'art moderne (Matisse,

\footnotetext{
${ }^{72}$ Il fut amené pour la première fois dans cette abbatiale à l'âge de 5 ans, par sa mère très pieuse, juste après la mort de son père. Il y est revenu vers 14 ans et fut «bouleversé par cette nef, la plus haute de l'art roman, cet édifice massif allié à tant de grâce. C'est là que j'ai décidé que l'art serait au centre de ma vie. ", "Conques est le lieu de mes premières émotions artistiques » (dans « Pierre Soulages, la lumière de l'inattendu », Sabine Gignoux, La Croix, 10 octobre 2009 et Christian Heck, Pierre Soulages, Jean-Dominique Fleury, Conques : les vitraux de Soulages, 1994, Paris, éd. Seuil).
} 
Derain, Picasso, Braque Miró, Léger...) furent eux aussi fascinés par ces œuvres au point de s'en laisser inspirer.

Ce que révélait habilement en 2016-2017 l'exposition «Picasso roman » au Museu Nacional d'Art de Catalunya à Barcelone, organisée conjointement avec le musée national Picasso-Paris. Elle faisait dialoguer une quarantaine d'œuvres du peintre cubiste (huiles, dessins, céramiques et sculptures) avec les œuvres médiévales du musée de Catalogne pour en capter les affinités. Une sélection de documents inédits des archives de Picasso appartenant au musée parisien dévoilait son intérêt pour l'art roman.

Deux dates surtout marquèrent sa relation avec l'art médiéval : 1906, où il s'installa plusieurs mois dans le village de Gósol (Pyrénées, France) alors que son œuvre opérait un retour à un certain primitivisme, en réaction à ce qu'il était convenu d'appeler la "période rose » antérieure, que lui-même qualifiait de «sentimentale »; et 1934, où il visita les collections d'art roman (qui constituent aujourd'hui le fonds du musée national d'art de Catalogne) la veille de son départ définitif du pays.

Suite à sa visite, Picasso parla d'un musée «unique au monde et indispensable pour connaître l'origine de l'art occidental», « une leçon pour les artistes modernes ». Et la presse relatait: «En passant d'une salle à l'autre, devant ces fragments incomparables de l'art primitif catalan, Picasso en admirait la force, l'intensité et la manière, la sûreté de vision et d'exécution, l'aplomb et la conviction avec lesquels la main de l'artiste inconnu avait exprimé dans ces panneaux muraux les idées ainsi que les sentiments qui lui occupaient l'esprit».

Les archives présentées (images romanes, cartes postales, correspondances, ouvrages et revues) confirment l'intérêt que l'artiste a toujours maintenu pour l'art médiéval. On ne peut cependant parler d'influence directe car Picasso sut dépasser les modèles originaux. Il semblait reconnaitre dans le roman la préexistence de solutions plastiques qui s'appliquent aussi aux problématiques de la création contemporaine. Il s'orienta alors vers des formes simplifiées et des compositions nonfiguratives. Ses personnages se font plus schématiques et hiératiques, ses toiles plus symboliques et teintées de mysticisme.

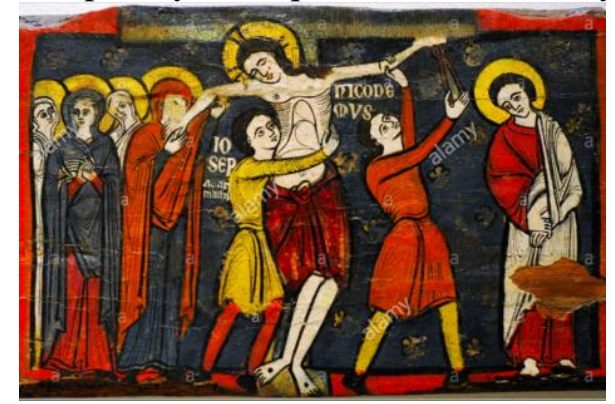

ill. 17 Détail de la descente de Croix, 1192-1220

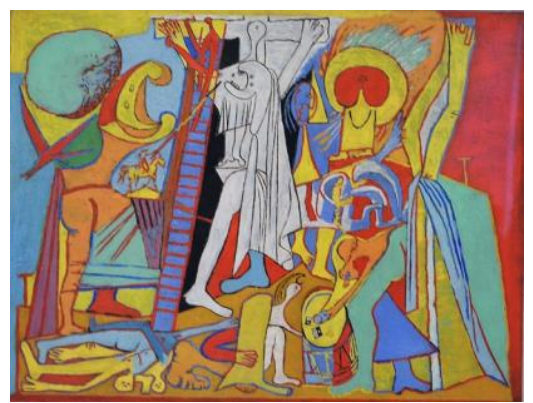

ill. 18 Pablo Picasso, Crucifixion, 1930 
Le thème de la crucifixion par exemple, très présent dans l'art roman, préoccupa Picasso en différents moments de sa vie, surtout de 1930 à 1937. On peut rapprocher les crucifixions désarticulées de Picasso des crucifixions articulées du roman, et tout spécialement l'ensemble sculptural et pictural des « descentes de la croix » conservées par le musée (ill. 17, 18). De même le sujet de la tête de mort (ou crâne), très présent dans la collection romane du musée, trouve écho dans le thème transversal de la mort chez Picasso, spécialement au travers de masques et de têtes de mort humaines ou animales.

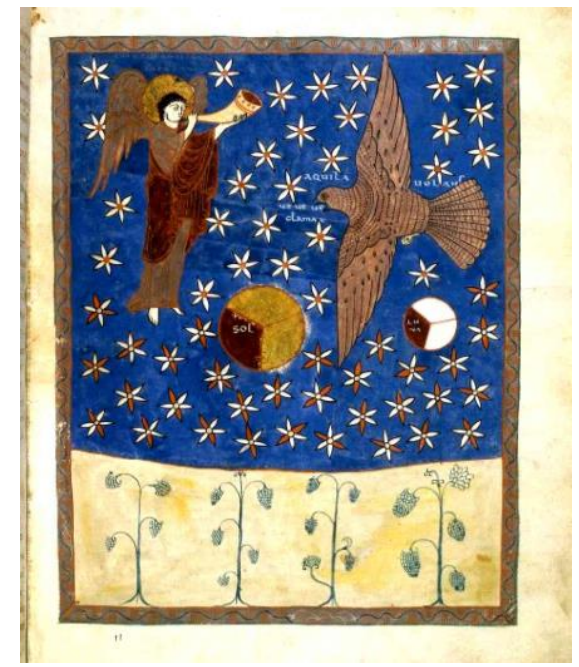

ill. 19 Apocalypse de Saint Sever, « La Quatrième trompette », folio 141

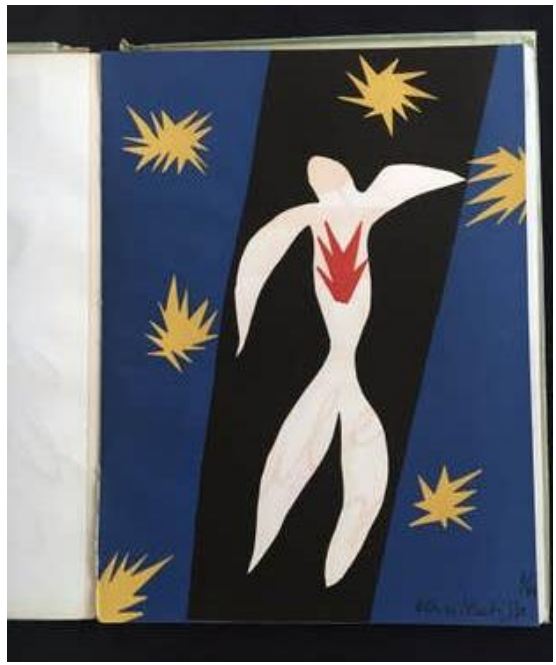

ill. 20 Frontispice revue Verve, n43, 1945 (Matisse, La Chute d'Icare)

De même, Matisse admira le manuscrit de l'Apocalypse de Saint Sever, déjà évoqué (ill. 1, 2 et 19, 20). Lorsqu'il fut édité en fac-similé en 1947 chez son éditeur Tériade, il se souvient avoir vu ce travail en cours tandis qu'il allait vérifier l'impression de son album Jazz. Matisse connaissait déjà sans doute très bien cette œuvre : il fit sa première couverture de Verve en 1933, dans un numéro où étaient parues des reproductions de l'Apocalypse de Saint-Sever. Et cette œuvre fut exposée en 1937 à la Bibliothèque nationale.

À neuf cents ans d'intervalle, ces deux livres ont eu une influence considérable sur leur époque : les gouaches découpées de Matisse marquèrent profondément la peinture contemporaine, alors que le Beatus de Saint-Sever fut l'une des sources majeures de la sculpture romane. ${ }^{73}$

\footnotetext{
${ }^{73}$ D'après Émile Mâle, L'Art religieux du XIIe siècle en France, Paris (1953), 1966, p. 45 ; cf. chap. I, II, XI.
} 
C'est seulement à partir des années 1920 que ce manuscrit a véritablement commencé à être regardé et étudié, notamment par Émile Mâle et Georges Bataille. Ce dernier en avait reproduit quelques pages dans le numéro 2 de la revue Documents en $1929 .{ }^{74}$ Picasso se souvenait bien de ce numéro puisque le 7 février 1930, il peint un petit tableau aux couleurs violentes, une Crucifixion (ill. 18), après avoir lu l'article consacré à ce manuscrit, illustré par six reproductions en noir et blanc.

D'où la juste expression de Francastel en 1946 sur certaines «affinités sentimentales » à travers les âges entre la grande famille d'artistes de l'art roman et celle de l'art moderne, au service d'un monde à rebâtir au lendemain de la Seconde Guerre.

\section{Liste et source des illustrations :}

ill. 1, 2 Stephanus Garsia et deux anonymes, Apocalypse de Saint Sever (ou Le Beatus de Saint Sever), mi-XI ${ }^{\mathrm{e}}$ siècle, $36,7 \mathrm{~cm} \times 28,6 \mathrm{~cm}, 296$ folios reliés, Bibliothèque Nationale de France, Paris

ill. 3 Albert Gleizes, Crucifixion, 1935, huile sur toile, 137 x $92 \mathrm{~cm}$, MBA Dijon (photo V. Buisine) - @ Adagp, Paris, 2021

ill. 4 Albert Gleizes, Les Evangiles, 1939-1941, papier gouache, $58.5 \mathrm{~cm} \mathrm{x} 44 \mathrm{~cm}$, collection particulière - @ Adagp, Paris, 2021

ill. 5 Albert Gleizes, Autorité spirituelle et pouvoir temporel, 1939-1940, huile sur toile, 336 x $203 \mathrm{~cm}$, musée des Beaux-Arts de Lyon - @ Adagp, Paris, 2021

ill. 6 Roger Bissière, Figure debout, 1937, huile sur carton, 143 x $25 \mathrm{~cm}$, musée d'Unterlinden, Colmar, France (photo V. Buisine) - ๑ Adagp, Paris, 2021

ill. 7 Roger Bissière, Grande Figure, 1937, 163 x 45 cm, collection particulière, Paris (photo V. Buisine) - @ Adagp, Paris, 2021

ill. 8 Roger Bissière, Joueuse de guitare, 1946-1947, huile sur papier marouflé sur panneau, MNAM Paris (photo V. Buisine) - @ Adagp, Paris, 2021

ill. 9 Roger Bissière, Grande cathédrale, 1947, huile sur papier marouflé sur toile, 193 x 95 cm, collection particulière, Paris (photo V. Buisine) - @ Adagp, Paris, 2021

ill. 10 Roger Bissière, Soleil, 1945-1946, tapisserie (morceaux de tissus divers : jute, coton, laine...), 170 x $245 \mathrm{~cm}$, musée de l'abbaye Sainte-Croix, Les Sables-d'Olonne, France (photo V. Buisine) - @ Adagp, Paris, 2021

ill. 11 Jean Lurçat, L'Apocalypse, 1947, tapisserie en laine, 453 x $1240 \mathrm{~cm}$, église Notre-Dame-de-Toute-Grâce, plateau d'Assy (Passy, France), tissée à Aubusson (photo V. Buisine) - @ Adagp, Paris, 2021

ill. 12 Jean Bertholle, Glorification, 1946, tissée en basse lisse, 230 x $175 \mathrm{~cm}$, collection particulière (photo V. Buisine) - ( Adagp, Paris, 2021

\footnotetext{
${ }^{74}$ D'après un texte écrit en 1971-1972 en anglais et publié sous le titre Two Books : the “Apocalypse of Saint-Sever” and Matisse's “jazz”, Los Angeles County Museum of Art, avrilmai 1972 ; repris dans Avigdor Arikha, Peinture et regard : Écrits sur l'art, 1965-2009, sous le titre «Matisse et l'Apocalypse de Saint-Sever : Beatus et Jazz », 1994 (rééd. 2011), Paris, Éditions Hermann, p. 13 à 29.
} 
ill. 13 Jean Bertholle, Devant d'autel (ou antependium), 1980, fragments de textiles cousus et brodés, $87 \times 111 \mathrm{~cm}$, collection particulière (photo V. Buisine) - ( ) Adagp, Paris, 2021

ill. 14 Alfred Manessier, Les Pèlerins d'Emmaüs, 1943, huile sur toile, 195,3 x 130,5 $\mathrm{cm}$, Centre Pompidou, Paris (photo V. Buisine) - ( ) Adagp, Paris, 2021

ill. 15 Alfred Manessier, Salve Regina, 1945, huile sur toile, 195 x 115 cm, Musée des beaux-arts, Nantes (photo V. Buisine) - ( ) Adagp, Paris, 2021

ill. 16 Pierre Soulages, quatre vitraux vus de l'intérieur, abbatiale Sainte-Foy de Conques, 1994 (photographie) - (C) Adagp, Paris, 2021

ill. 17 Détail de la descente de Croix, tempera sur bois, 1192-1220, origine catalane, Musée national d'art de Catalogne, Barcelone (Espagne)

ill. 18 Pablo Picasso, Crucifixion, 1930, tempera sur bois, origine catalane, Musée national d'art de Catalogne, Barcelone (Espagne) - (C) Succession Picasso 2021

ill. 19 Apocalypse de Saint Sever, "La Quatrième trompette », mi-XI ${ }^{\mathrm{e}}$ siècle, folio 141, Bibliothèque Nationale de France, Paris

ill. 20 Frontispice de la revue Verve, ${ }^{\circ}$ 13, 1945 (reproduction de Henri Matisse, La Chute d'Icare, été 1943, 35 x 26,2 cm - photo V. Buisine) - (C) Succession H. Matisse 2021

\section{Bibliographie}

\section{Ouvrages et catalogues d'exposition :}

Abadie, Daniel, Bissière, Neuchâtel, Éditions Ides et Calendes, 1986

Bertrand Dorléac, Laurence, L'art de la défaite, 1940-1944, Paris, Seuil, 1993 (rééd. 2010, 2012) (notamment les pages 169 à 261) (L'art de la défaite 1993)

Bissière, Isabelle, Duval, Virginie et Lemoine, Serge (préface), Roger Bissière (1886-1964), catalogue raisonné (3 tomes), Neuchâtel, Editions Ides et Calendes, 2001

Bissière, Roger, T'en fais pas la Marie - écrits sur la peinture 1945-1964, textes réunis et présentés par Marrey, Baptiste Cognac, ssệ̂, Le Temps qu'il fait, 1994

Ceysson, Bernard, Fleck, Robert, Bissière, Paris, éd. Ceysson, 2010

Chastel, André et al., Henri Focillon, Centre Georges-Pompidou, Paris, 1986

Ramond, Sylvie, Brüggen, Viktoria von der, Bissière : "Pense à la peinture », catalogue exposition Colmar, musée d'Unterlinden, Lyon, Fage, 2004

Massenet, Michel, Albert Gleizes, Paris, Somogy, 1998

Robbins, Daniel, «Albert Gleizes : son expression de la foi et de la raison dans la peinture moderne », dans Albert Gleizes 1881-1953, catalogue exposition rétrospective, MNAM, Paris, 15 déc. 1964-31 janvier 1965

Vollerin, Alain, Le groupe Témoignage de Lyon (1936-1940), Lyon, éd. Mémoire des arts, 2001

Bissière, Le rêve d'un sauvage qui aurait tout appris, musée Picasso d'Antibes, château Grimaldi, 1999

Focillon, catalogue exposition MBA de Lyon et INHA (Institut National de l'Histoire de l'Art, Paris), 2004

Groupe Témoignage, 1936-1943, cat. expo., Lyon, MBA, 1976 
L'Art en guerre. France 1938-1947, cat. expo. MAM ville de Paris, oct. 2012-fév. 2013, 2012

Le Poids du monde. Marcel Michaud (1898-1958), sous la direction de Laurence Berthon, Sylvie Ramond et de Jean-Christophe Stuccilli, Lyon, MBA, 22 octobre 2011 au 23 janvier 2012, Lyon, Fages éditions, 2011

Marcel Michaud, Lyon, 1933-1958, Stylclair, Groupe Témoignage, Galerie Folklore, texte de Bernard Gavoty, Espace Lyonnais d'Art Contemporain, Lyon, 1989

Montparnasse années 30 - Bissière, Le Moal, Manessier, Étienne-Martin, Stahly \& les autres... Éclosions à l'Académie Ranson, Rambouillet, Palais du roi de Rome, Belgique, éd. Snoeck, 2010

Paris-Paris : 1937-1957 : arts plastiques, littérature, théâtre, cinéma, vie quotidienne et environnement, archives sonores et visuelles, photographie, catalogue d'exposition, Paris, éd. Centre Pompidou, 28 mai-2 nov. 1981 ( $2^{\mathrm{e}}$ éd. Paris, Gallimard, 1992)

\section{Articles :}

Dossier «L'art roman au $\mathrm{XX}^{\mathrm{e}}$ siècle », revue Arts sacrés, $\mathrm{n}^{\circ} 2$, Paris, éd. Faton, nov.-déc., 2009

Gleizes, Albert, conférence «Art et religion» (21 mars 1931 à Paris), Art et religion, Art et science, Art et production, Chambéry, Présence, 1970

Gleizes, Albert, « Recherche du sacré », L'Art sacré, n4-5, avril-mai 1947, pp. 125 127

Greff, Jean-Pierre, «Le thème de l'art mural dans les années trente », Asse, Bazaine... Maquettes des vitraux de la cathédrale et cuvres récentes, musée Saint Dié, 16 avril-29 mai 1988, p. 14-18

Harambourg, Lydia, article «Roger Bissière », dans dictionnaire L'École de Paris 1945-1965, 1993

Le Poids du Monde (du groupe Témoignage), revue $\mathrm{n}^{\circ} 1$ à 4, 1937 à 1940 (Bibliothèque Kandinsky, Centre de documentation et de recherche du MNAM / Cci, Centre G. Pompidou, Paris)

Zodiaque, numéro spécial «présence d’Albert Gleizes », n6-7, $2^{\mathrm{e}}$ année, 1952

\section{Travaux universitaires :}

Arbour-Mayrand, Rose-Marie, Bissière et la tradition de la peinture, thèse de $3^{\mathrm{e}}$ cycle, Université Paris Sorbonne, 1973, René Jullian (directeur)

Blanchon-Gaudin, Laëtitia, Regard sur Témoignage, mémoire de master 1 d'histoire de l'art, Lyon, Université Lumière Lyon 2, 2010, Annie Claustres (directrice)

Charvier, Alexandra, L'Académie Ranson, creuset des individualités artistiques, 1919-1955, mémoire de maîtrise, univ. Paris 1, Panthéon-Sorbonne, Paris, 2003, Guitemie Maldonado (dir.)

Gavoty, Bernard, Marcel Michaud, personnalité mythique du milieu de l'art Lyonnais dans les années 1930-1950, mémoire de maîtrise d'histoire de l'art, Mady Menier (dir.), Université Lumière, Lyon II, 1989

Goudal-Nicollier, Sandrine, L'Académie Ranson, 1908-1918, mémoire de $3^{\mathrm{e}}$ cycle, École du Louvre, Paris, 1997, Guy Cogeval (dir.) 
Thomas de Closmadeuc-Perrot, Françoise, Témoignage, un groupe d'artistes "lyonnais » entre 1936 et 1940, mémoire de maîtrise d'histoire de l'art, université Lumière Lyon 2, 1998, Gilles Chomer (dir.)

Stahl, Fabienne, L'œuvre-sacré des artistes de "tradition française », mémoire de maîtrise, Strasbourg, Université des sciences humaines, Institut d'Histoire de l'art, 1996, Christine Peltre (dir.)

André Guégan, «Bertholle... d'une saveur médiévale », La Bretagne à Paris, 25 janv. 1980

\section{Archives :}

Boîte «Témoignage » dans les archives de Jean Bertholle, Paris

Fonds d'archives Marcel Michaud sur le groupe Témoignage, MBA Lyon

\section{Filmographie :}

Le Comte, Daniel, Le groupe Témoignage, Accord CLC ${ }^{75}$ productions, 2000, couleurs, $52 \mathrm{mn}^{76}$

Pavlowitch, Paul et Annie, Roger Bissière ou le regard du cœur, 18 mn, Écran Sud, Toulouse, 1990

\footnotetext{
${ }^{75}$ Compagnie Lyonnaise de Cinéma.

${ }^{76}$ Prix du documentaire historique au $25^{\mathrm{e}}$ Festival international du film d'art à l'UNESCO.
} 